\title{
Local Marangoni number at the onset of hydrothermal waves
}

\author{
Miguel Angel Pelacho, ${ }_{1}^{1}$ Angel Garcimartín, ${ }^{1}$ and Javier Burguete ${ }^{1,2}$ \\ ${ }^{1}$ Departamento de Física y Matemática Aplicada, Universidad de Navarra, E-31080 Pamplona, Spain \\ ${ }^{2}$ CEA-Service de Physique de l'Etat Condensé, Centre d'Etudes de Saclay, 91191 Gif-sur-Yvette, France
}

(Received 23 December 1999)

\begin{abstract}
We report the results of an experiment in which a layer of fluid, placed in a container open to the air, is subjected to a horizontal temperature difference $\Delta T$. The fluid height $h$ is kept constant and both the horizontal temperature difference and the horizontal dimensions of the fluid layer are changed. In this configuration, when $\Delta T$ goes beyond a certain threshold, waves propagating from the cold to the hot side appear (hydrothermal waves) with a determined group velocity. We study the influence of the container geometry on these waves. Close to the threshold, they are spatially localized near the hot side and a local Marangoni number is defined to describe this behavior. If $\Delta T$ is further increased, the waves fill the whole fluid layer. We also find an agreement between our experimental results and theoretical works and simulations.
\end{abstract}

PACS number(s): 47.20.Dr, 47.54. $+\mathrm{r}$

\section{INTRODUCTION}

The results of an experiment in which a horizontal temperature gradient is imposed on a fluid layer open to the air are presented in this paper. An analytical study of these systems is difficult to carry out due to the existence of boundaries. The boundary conditions and the presence of vertical boundary layers complicate any realistic theoretical study. In fact, some divergences are found between theoretical and experimental works. In the former, the layer is often assumed to have an infinite horizontal length and a constant temperature gradient. As a result, the analytical calculus and the simulations are feasible but models are not as close to experiments as one would wish.

In this system, i.e., a fluid layer heated from the side, a basic flow appears as soon as a temperature difference $\Delta T$ $>0$ is applied between the hot and the cold walls. It consists in a roll with the axis perpendicular to the gradient. A surface flow is created from the hot to the cold side, and a return flow appears at the bottom. This basic flow, homogeneous in the direction perpendicular to the temperature gradient, can be destabilized mainly by the action of buoyancy and thermocapillary forces. When $\Delta T$ is increased over a certain threshold $\left[\Delta T_{c}(h)\right]$, the basic flow destabilizes and hydrothermal waves appear via a supercritical bifurcation [1]. Two nondimensional numbers evaluate the magnitude of these forces versus the viscous dissipation. The Marangoni number

$$
\mathrm{Ma}=\frac{\left|\frac{\partial \sigma}{\partial T}\right| \beta h^{2}}{\rho \nu \kappa}
$$

is the ratio between thermocapillary forces and viscous dissipation, and the Rayleigh number

$$
\mathrm{Ra}=\frac{g \alpha \beta h^{4}}{\nu \kappa}
$$

is the ratio between buoyancy forces and viscous dissipation ( $g$ is the gravitational acceleration, $\beta$ the horizontal temperature gradient, $\alpha$ the thermal expansion coefficient, $\rho$ the fluid density, $\nu$ the kinematic viscosity, $\kappa$ the thermal diffusivity, and $\sigma$ is the interfacial tension).
The first linear stability analysis of this system was carried out by Smith and Davis [2] considering fluids with different Prandtl numbers $(\operatorname{Pr}=\nu / \kappa)$. Only thermocapillary forces were taken into account, neglecting particularly the role of gravity. They found that a pair of traveling waves appears superimposed to the basic flow for a particular value of the temperature gradient, traveling at a certain angle with respect to it. The dependence of some properties of the waves (frequency, wave number, and angle of propagation) on the Pr number was found. A few years later, Smith [3] explained in a qualitative way the instability mechanism giving rise to these hydrothermal waves: a perturbation on the temperature field amplified by the interaction with the velocity field.

Buoyancy forces, however, were not taken into account. They were included in the works of Gershuni et al. [4], Parmentier et al. [5], and Mercier and Normand [6]. In the latter, a transition from oscillatory modes towards steady modes is found for a certain fluid height, as was observed by Daviaud et al. [7]. Depending on the value of the fluid height, one of the two forces (buoyancy or thermocapillarity) dominates over the other one. As hydrothermal waves are thermocapillary instabilities, it is reasonable to choose a fluid layer of small depth so that thermocapillary forces dominate over buoyancy. Many experiments have been performed in shallow layers, with rectangular or annular containers and with similar or very different horizontal dimensions $[7,8,10$ 14]. In some of them, the influence of the side walls is obvious while in others it is not quite clear.

As it seems that the instability mechanisms for small or large Pr number are different [3], we expect to obtain both in a fluid with intermediate Pr number. In the range of Pr numbers from 4 to 30 , a rich variety of dynamical behaviors has been observed. In several experiments with $\operatorname{Pr} \simeq 30$ and various geometries $[8,10,11]$, traveling waves have been seen with their wave vector parallel to the temperature gradient. For fluids whose Pr number has a value between 4 and 15, the situation is different. When the distance between the hot and cold walls, $l_{x}$ (referred to as end walls in the following), was much shorter than the distance between the lateral walls, $l_{y}$ (referred to as side walls), an angle of propagation (angle between the wave vector and the temperature gradient) close 
to $90^{\circ}$ was measured $[7,12]$. However, if $l_{x}$ is similar to $l_{y}$, the angle of propagation has a value between $40^{\circ}$ and $50^{\circ}$ [13] or $23^{\circ}$ [14].

The first objective of our work is to find out the influence of the geometry on the hydrothermal waves and try to match the results of those experiments, providing an explanation for their physical behavior. To do this, we made a container with variable $l_{x}$ and $l_{y}$ in order to try to recover the results of previous experiments (for $l_{x} \ll l_{y}, l_{x} \simeq l_{y}, l_{y} \ll l_{x}$ ) and reconcile the seemingly divergent observations.

As hydrothermal waves are originated by thermocapillary stresses, our experiment is performed in a shallow layer with constant height $h=1.5 \mathrm{~mm}$, in which the Marangoni number is more than four times bigger than the Rayleigh number $(\mathrm{Ra} / \mathrm{Ma} \leqslant 0.25)$ so that thermocapillary effects are presumed to dominate over the buoyancy ones.

The second aim of this study concerns the Marangoni number at the threshold of the waves. There is often a discrepancy between the theoretical value of the Ma number and the value obtained in experiments. In all the experiments the measured Ma number is bigger than the analytical one, calculated taking a constant horizontal temperature gradient. On the contrary, in experimental systems end walls are unavoidable and the temperature gradient changes abruptly near them, yielding a critical global Ma number bigger than the theoretical one. This global Ma number is calculated from the temperature difference between the end walls. As we will explain, one needs to consider the locally measured $\mathrm{Ma}_{L}$ number as the parameter of the system instead of a global Ma number.

This paper is divided in the following sections. Section II contains the experimental procedures. In Sec. III we show the results concerning the influence of the geometry, the measured local Marangoni number, and the amplitude of the hydrothermal waves. Finally, in Sec. IV we state some conclusions.

\section{EXPERIMENTAL PROCEDURE}

A silicon oil of kinematic viscosity $\nu=0.65 \mathrm{cSt}$ and Pr $=10$ is poured into a nylon container with the upper surface open to the air. The height of the fluid is set at $1.5 \mathrm{~mm}$ using a micrometer with an accuracy of $10 \mu \mathrm{m}$. Owing to the fast evaporation of the fluid a cover was needed to reduce it as much as possible. We measured that in two hours (time enough to make one experimental run) the height decreased by about $2 \%$. In the range of the applied temperatures the physical properties of the fluid do not suffer a great change (the Boussinesq approximation is valid).

The container (Fig. 1) has two copper blocks at opposite sides and two plexiglass blocks placed perpendicularly to the copper blocks. The distance between the copper end walls is $l_{x}$ and the distance between the plexiglass side walls is $l_{y}$. One of the copper blocks is movable so that $l_{x}$ can be changed from $41 \mathrm{~mm}$ to $100 \mathrm{~mm}$. Both plexiglass blocks are also movable in the same range. A temperature $T_{+}$is imposed on one of the copper walls $(x=0)$ and a temperature $T_{-}<T_{+}$on the other wall. They are approximately symmetrical to the room temperature $\left(\simeq 22^{\circ} \mathrm{C}\right)$. Thus a temperature gradient is established along the $x$ axis. The mass of the copper pieces is big enough to ensure that their temperature
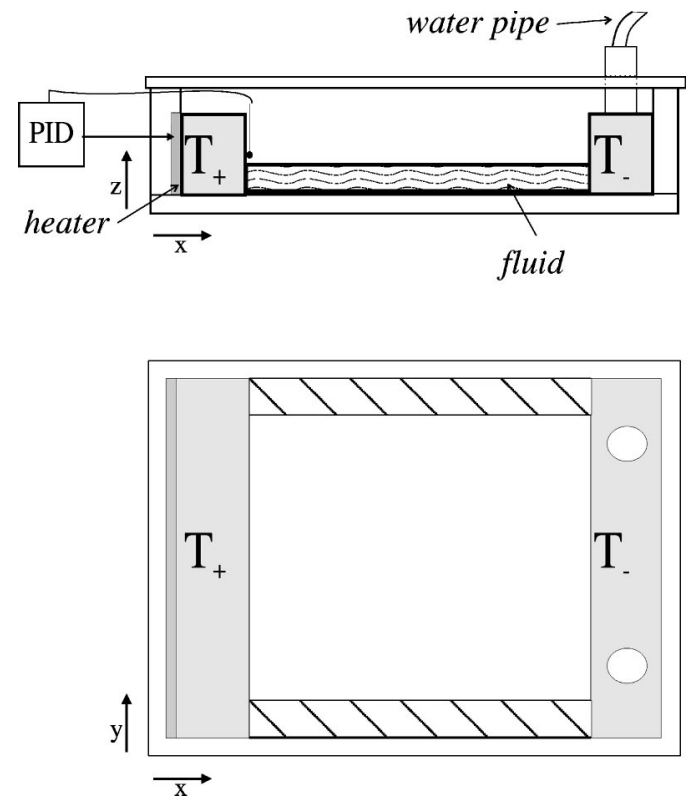

FIG. 1. Side view (above) and top view (below) of the container. Temperature $T_{-}$is stabilized by a regulated thermal bath and $T_{+}$by a PID control acting on a heating resistance. The height of the fluid layer is $h=1.5 \mathrm{~mm}$.

is not disturbed by the temperature oscillations of the fluid. At the bottom of the container an aluminum sheet provides an approximately linear temperature profile.

The external parameters are therefore the horizontal temperature difference $\left(\Delta T=T_{+}-T_{-}\right)$and the distances $l_{x}$ and $l_{y}$. In order to measure $T_{+}$and $T_{-}$two thermocouples are stuck on the inner side of the copper blocks near the fluid. A thermocouple is also placed above the fluid at the middle of the cell to monitor the room temperature.

The control of $T_{+}$and $T_{-}$was achieved as follows. Cool water coming from a thermostatic bath $\left( \pm 0.01^{\circ} \mathrm{C}\right)$ is circulated inside one of the copper blocks. On the other copper block an electrical heater, glued to its outer side, is controlled by means of a PID loop (see Fig. 1). The amplitude of temperature oscillations in both sides is smaller than $0.02^{\circ} \mathrm{C}$.

A shadowgraph of the fluid is captured by a camera and stored in the computer. Spatiotemporal diagrams are made in order to obtain the main physical properties of the waves: frequency, wave number, and angle of propagation. An explanation of this technique can be found in Ref. [8]. Other characteristics of the waves, such as the group velocity, have been obtained using the techniques described in Ref. [9].

In order to measure the temperature profile in the fluid, a thermocouple is introduced inside it and moved by an $x y z$ motorized micrometer. It is programmed and controlled by a computer to get the temperature data at different space and time intervals depending on whether it is moving in the $x, y$, or $z$ axis. Between 50 and 100 temperature data points, depending on the value of $l_{x}$, are registered to obtain a good temperature profile. The time spent in each measurement is much longer than the response time of the thermocouple $\tau_{\text {th }}<0.2 \mathrm{~s}$. A noninvasive method was also used to measure the temperature at the surface: an infrared sensor placed 10 $\mathrm{mm}$ above the fluid surface. 


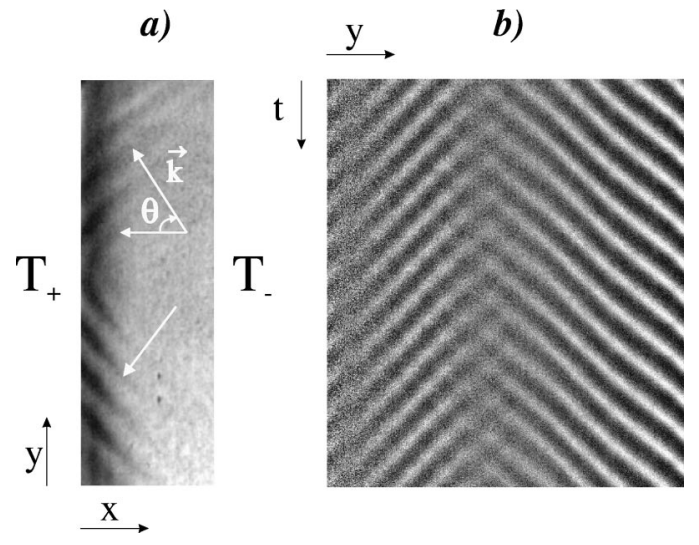

FIG. 2. (a) Shadowgraph image of the oblique traveling waves appearing near the hot end; (b) picture of a spatiotemporal diagram obtained by taking a vertical line in the $y$ direction at regular intervals. In this case the applied temperature difference is $\Delta T_{c}$ $=10^{\circ} \mathrm{C}$ and the distances $l_{x}=75 \mathrm{~mm}, l_{y}=100 \mathrm{~mm}$.

\section{RESULTS}

When $\Delta T=T_{+}-T_{-}$is increased, the basic flow destabilizes and a pair of oblique traveling waves appears superimposed to the basic flow. Waves, as observed by shadowgraphy, appear near the hot side in a region close to the side walls. When $\Delta T$ is further increased, the region where the waves exist grows up to fill the container.

Each wave propagates with a wave vector $\vec{k}$ as shown in Fig. 2 (left side), pointing towards the hot side, and forming an angle $\theta$ with the temperature gradient $\vec{\nabla} T$. We will refer to the wave propagating in the positive (negative) $y$ direction as $\vec{k}^{+}\left(\vec{k}^{-}\right)$. Each one of these waves has the same wavenumber modulus $|k|$, with opposite $y$ component, $k_{y}^{+} \simeq$ $-k_{y}^{-}$, and consequently with opposite angle of propagation $\theta^{+} \simeq-\theta^{-}$. At the right side of Fig. 2 a spatiotemporal diagram is shown as obtained for a line parallel to the $y$ axis. In Sec. III A we will explain this result in more detail.

The first step is to characterize the thermal behavior of the fluid layer and, in particular, to evaluate the temperature gradient $\vec{\nabla} T(x, y, z)$ at each point. The results explained in the following apply to all the geometrical configurations $\left(l_{x}, l_{y}\right)$. In order to see if the local horizontal gradient depends on $z$, several vertical profiles were obtained at different places $(x, y)$ moving the thermocouple along the $z$ axis. Then we calculated the variation of the horizontal temperature gradient at different depths. This variation was about $2 \%$, so the horizontal temperature gradient is approximately the same, regardless of $z$, except near the end walls. For $h>3 \mathrm{~mm}$, the temperature field becomes $z$-dependent [16] and other effects must be taken into account.

To obtain the temperature field in a horizontal plane, a thermocouple is introduced in the fluid at different depths $z$ and moved in the $x-y$ plane. Along the $x$ direction, far from the end walls the temperature profile is nearly linear, so the $x$ component of the gradient is approximately constant. Near the end walls, the temperature varies abruptly [see Fig. 3(a): typically $3{ }^{\circ} \mathrm{C}$ near each end wall, in the regions $x$ $<10 \mathrm{~mm}$ and $x>90 \mathrm{~mm}$, and thus the central gradient $|\vec{\nabla} T|_{x=l_{x} / 2}$ is smaller than $\Delta T / l_{x}$.

Along the $y$ direction, the temperature remains stable in
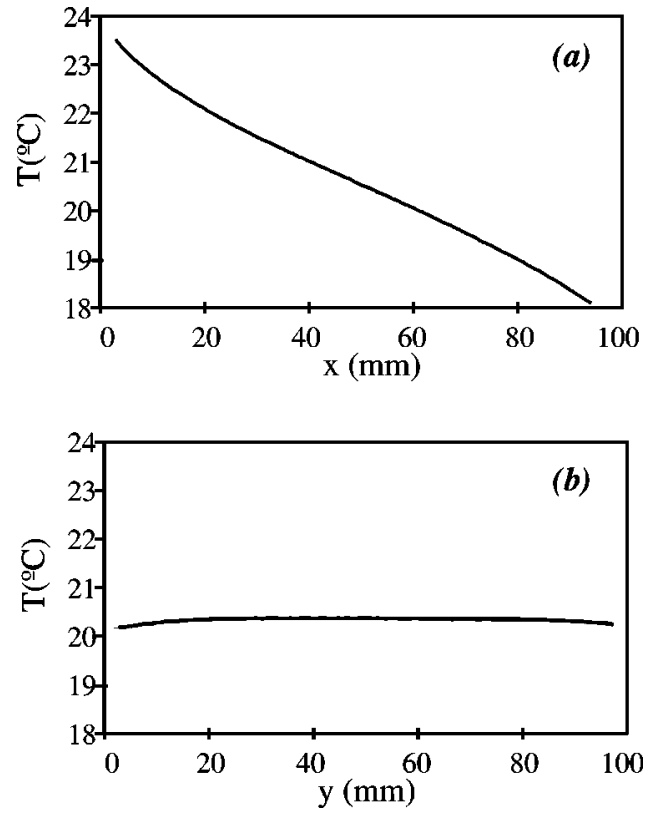

FIG. 3. Horizontal temperature profiles at $z=0.5 \mathrm{~mm}$ from the bottom, for $l_{x}=100 \mathrm{~mm}$ and $l_{y}=100 \mathrm{~mm}$. (a) Temperature profile along $x$. The curve contains more than 80 data points obtained when waves appear, for $\Delta T \approx \Delta T_{c}$. For $x<10 \mathrm{~mm}$ and $x>90 \mathrm{~mm}$ the profile is not more linear due to the presence of lateral boundary layers in both end walls. (b) Temperature profile along the $y$ axis. The influence of the side walls is noticeable at $y=15 \mathrm{~mm}$ near this side.

the central region, far from the side walls, and so the gradient has only an $x$ component, i.e., $\partial_{y} T \simeq 0$. Near the two side walls, the temperature decreases $0.15^{\circ} \mathrm{C}$ in a zone about $l_{d}$ $\simeq 15 \mathrm{~mm}$ wide [Fig. 3(b)]. For small $l_{y}$, the instability observed is different (thermocapillary waves are not seen).

We measured the critical temperature difference $\Delta T_{c}$ at the appearance of the waves using two methods to observe them. First, with spatiotemporal diagrams we can distinguish the traveling waves near $\Delta T_{c}$. However, previous works [1] have shown that shadowgraphy can induce an overestimate of the threshold. To avoid this, a second method is used, recording the temporal evolution of the fluid temperature with thermocouples and an infrared sensor. The threshold is determined by the appearance of oscillations in the temporal signal. The minimum detectable temperature amplitude for which we found oscillations is $0.02{ }^{\circ} \mathrm{C}$, and there was no noticeable difference in the experimental resolution on the threshold values obtained with both methods.

In our case, the experimental parameters are homogeneous in the $y$ direction, and as expected the wave dynamics does not show any change along this axis. A dynamical behavior can be observed in the $x$ direction if we go beyond the threshold. As has been shown in previous works, the waves appear via a supercritical bifurcation [1], and then near the threshold the wave can be described by

$$
S(y, t)=A(y, t) \exp \left[i\left(k_{y} y-\omega_{0} t\right)\right]+\text { c.c. },
$$

where $A$ obeys a complex Ginzburg-Landau equation:

$$
\partial_{t} A+v_{y}^{\mathrm{gr}} \partial_{y} A=\epsilon A+\xi_{0}^{2}\left(1+i c_{1}\right) \partial_{y y} A-g\left(1+i c_{2}\right)|A|^{2} A,
$$




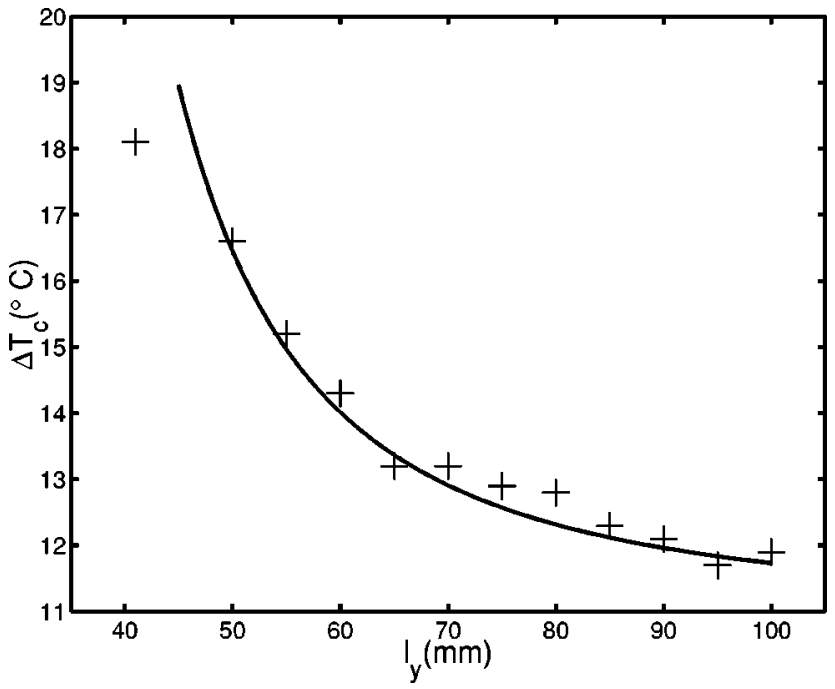

FIG. 4. Thresholds of hydrothermal waves for $l_{x}=100 \mathrm{~mm}$ and variable $l_{y}(+)$. Solid line: Fit of the experimental data (see explanation in the text). For $l_{y}=41 \mathrm{~mm}$ a mixture of different instabilities has been observed.

where $v_{y}^{\mathrm{gr}}$ is the $y$ component of the group velocity, $\xi_{0}$ is the correlation length, $g$ is the saturation, and $\epsilon$ is the distance from the threshold [9]. This description will be used in the following.

\section{A. Influence of the container geometry}

Our first objective, as we have said, is to ascertain the influence of the container dimensions $\left(l_{x}, l_{y}\right)$ on the threshold of the waves. To do this we have changed these parameters separately, varying $l_{y}$ with $l_{x}=$ const, and after that varying $l_{x}$ with $l_{y}=$ const.

In Fig. 4 the influence of $l_{y}$ on $\Delta T_{c}$ is shown for a constant $l_{x}=100 \mathrm{~mm}$. It is clear that there is a damping effect when $l_{y}$ is very short. This was observed before in some simulations [15] and this can be interpreted as a consequence of the finite size of the cell. It is known that in finite-size systems $[18,19]$, the boundary conditions stabilize the basic state and the threshold increases, $\epsilon_{l_{y}}^{*}=\left(\xi_{0} \pi / l_{y}\right)^{2}$. In our system, the control parameter is the temperature gradient $\beta$ and we expect it to increase when $l_{y}$ decreases in the form $\beta$ $=\beta_{\infty}\left(1+\xi_{0}^{2} \pi^{2} / l_{y}^{2}\right)$, where $\beta_{\infty}$ corresponds to the threshold in an infinite layer. In Fig. 4, we show the threshold values $\Delta T_{c}$ as a function of $l_{y}$, and a fit $\Delta T_{c}\left(l_{y}\right)=\Delta T_{\infty}(1$ $+\pi^{2} \xi_{0}^{2} / s_{y}^{2}$ ), where $s_{y}=l_{y}-2 l_{d}$ is the effective $y$ dimension, $\Delta T_{\infty}=11^{\circ} \mathrm{C}$, and $\xi_{0}=6.5 \mathrm{~mm} \simeq \lambda_{y}$.

As mentioned before, for small $l_{y}$ other instability mechanisms can appear. This is in concordance with the results of Fig. 4, where the experimental data obtained for $l_{y}$ $=41 \mathrm{~mm}$ correspond to a mixture of different instabilities that grow before the hydrothermal waves appear.

In addition, the group velocity of these waves is not negligible (see below), which makes the instability convective at the threshold $\epsilon_{c}=0$ [20]. In finite-size cells we can observe only the waves when the absolute threshold $\epsilon_{a}>\epsilon_{c}$ is surpassed, thus yielding an overestimate of the threshold.

If $l_{x}$ is reduced maintaining $l_{y}$ constant at $100 \mathrm{~mm}$, the threshold $\Delta T_{c}$ decreases (Fig. 5). This was expected because

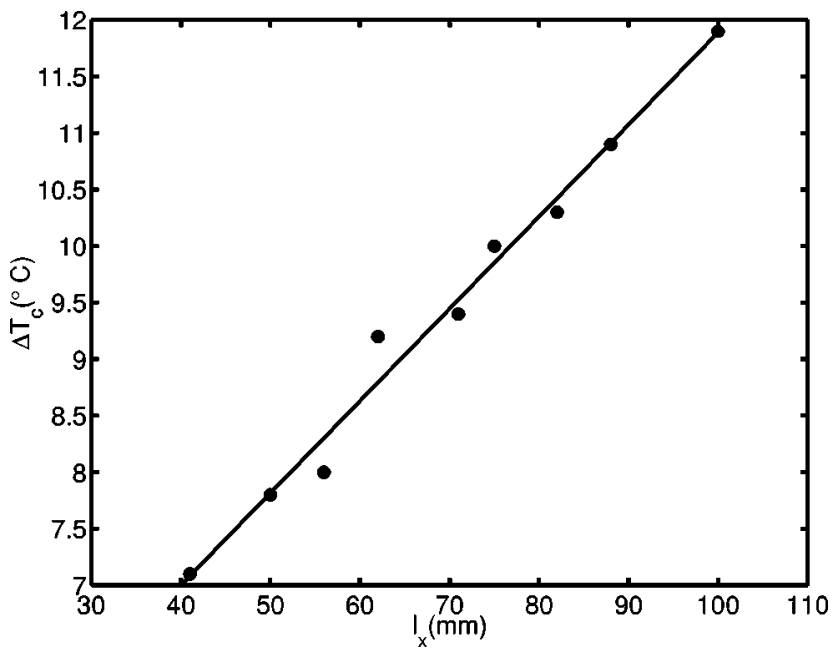

FIG. 5. Temperature difference at threshold of hydrothermal waves $\left(\Delta T_{c}\right)$, for $l_{y}=100 \mathrm{~mm}$, as a function of $l_{x}(\boldsymbol{O})$. Solid line: linear fit for the thresholds.

as $l_{x}$ is reduced, a smaller $\Delta T_{c}$ is needed to attain the same gradient $\beta$ and the same value of Ma.

Taking into account some experimental results related to the geometrical influence over the generation of traveling waves, an interesting general behavior can be set. One can observe a transition from the basic flow to a traveling waves pattern when the approximated linear relation between $l_{x} / l_{y}$ and $\Delta T_{c}$, shown in Fig. $6(+$ symbol), is reached. Actually we are studying the reasons for this behavior. In this picture, the results found by the authors of Refs. $[1,7,16]$ are compatible with our results. The discrepancy of the threshold found in the work of Ref. [13] is probably due to the difference between the thermal conditions at the bottom of the cell. In this latter case the bottom is not a good conductor and the temperature profile at the bottom is not linear (i.e., the temperature gradient is not constant), whereas in our ex-

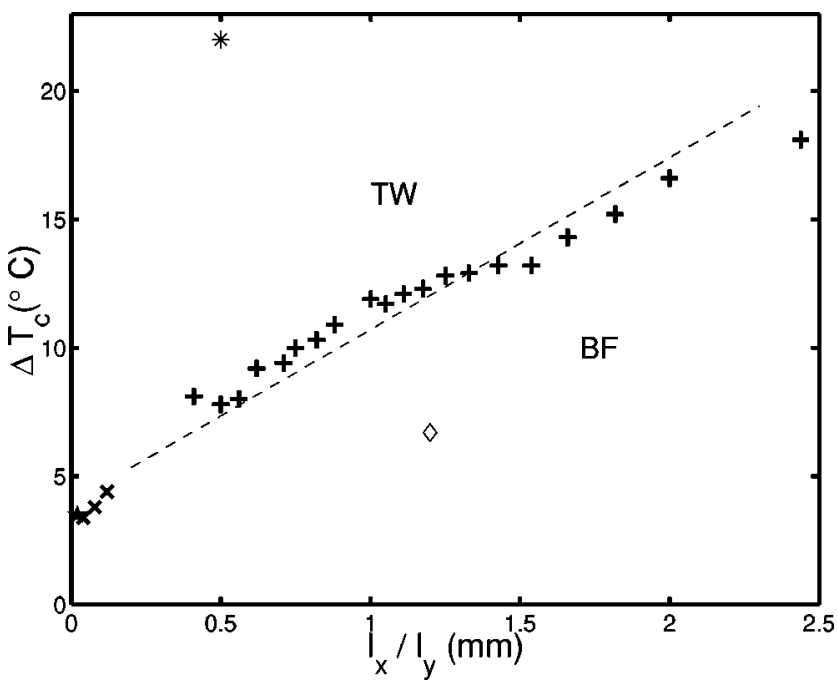

FIG. 6. Phase diagram for hydrothermal waves depending on the ratio $\left(l_{x} / l_{y}\right)$ (TW denotes traveling waves and BF denotes basic flow). + , data obtained in our experiment. Below these points only the basic flow is present and above them traveling waves appear. The rest of the points show the results of various experiments for the presence of traveling modes: $\times$ for Refs. [16,7], $\star$ for Ref. [1], $\diamond$ for Ref. [13], and * for the work of Ref. [12]. 

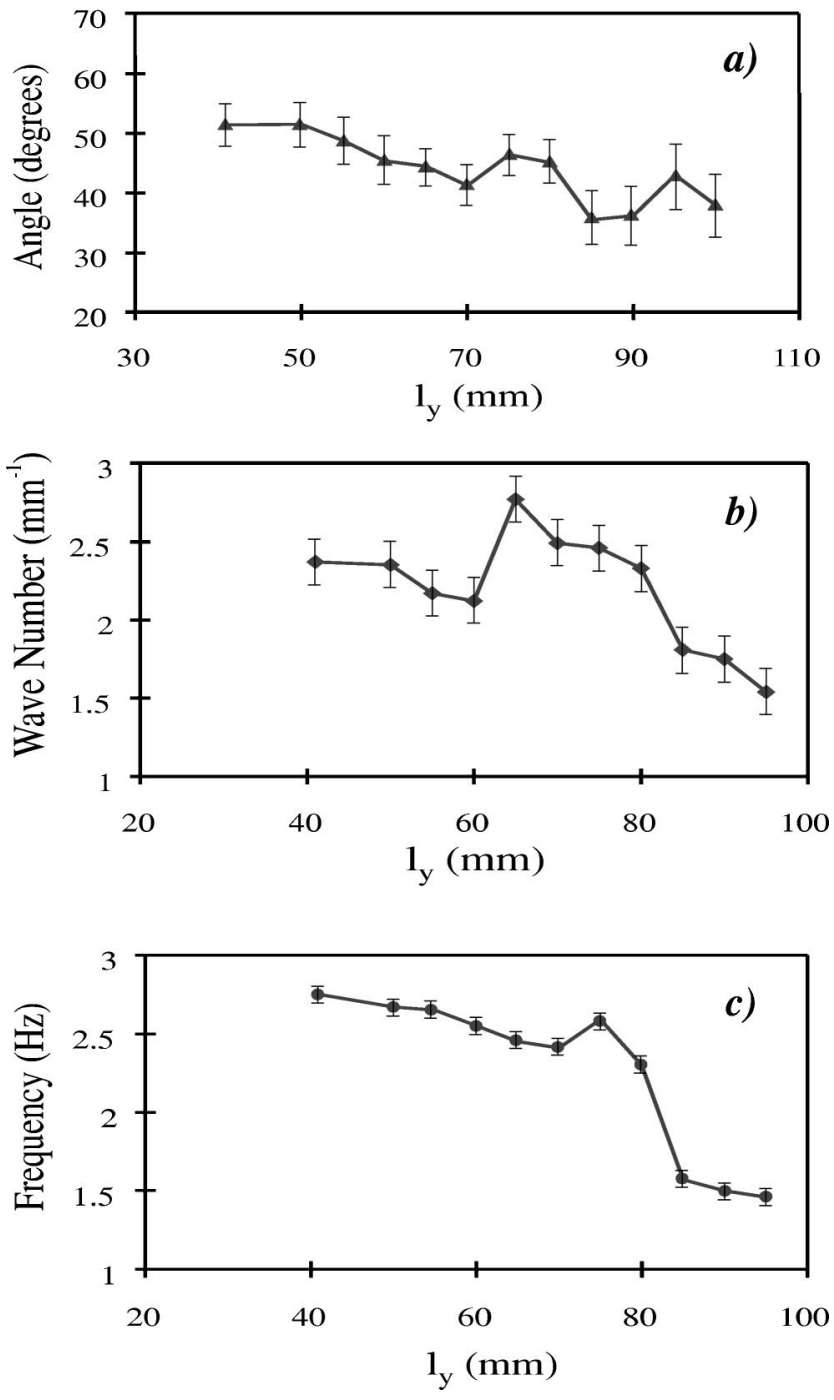

FIG. 7. Angle of propagation (a), modulus of the wave number (b), and frequency (c) for $l_{x}=100 \mathrm{~mm}$, as a function of $l_{y}$. The values are given at the threshold $\Delta T_{c}$ of the hydrothermal waves.

periment the conductor bottom provides a better aproximation to the theoretical analysis, which considers a constant temperature gradient. The traveling mode given by Ref. [12] corresponds to a fluid with high Prandtl number, $\operatorname{Pr}=17$, which can be the reason for the divergence.

From the spatiotemporal diagrams we can get the main physical properties of the waves (wave number $|\vec{k}|$, frequency $\omega$, and angle of propagation $\theta$ ) and their variation with the geometry. In the case $l_{x}=100 \mathrm{~mm}$ and variable $l_{y}$, the angle of propagation of the $k^{+}\left(k^{-}\right)$oblique wave increases (decreases) from $35^{\circ}\left(-35^{\circ}\right)$ to $52^{\circ}\left(-52^{\circ}\right)$ as $l_{y}$ decreases [Fig. 7(a)]. If $l_{x} \ll l_{y}$, the angle has a value slightly above $30^{\circ}$, which is contrary to what is found in other works $[7,12]$, namely $\psi \sim 90^{\circ}$, maybe due to the constraint in the $x$ direction of their container. Our value, however, agrees with the results obtained in [16] for the cases $l_{x}=20 \mathrm{~mm}$ and $l_{x}$ $=30 \mathrm{~mm}, l_{y}=250 \mathrm{~mm}$, and $h=1.5 \mathrm{~mm}$ : the angle obtained is $\phi=180-\psi=2.6 \mathrm{rad}$, thus $\psi \simeq 31^{\circ}$. If $l_{x}$ is similar to $l_{y}$, the angle has a value between $30^{\circ}$ and $40^{\circ}$, a result in agreement with Refs. $[13,14]$, where $l_{x}=60 \mathrm{~mm}$ and $l_{y}=50 \mathrm{~mm}$ for the first reference, and $l_{x}=30 \mathrm{~mm}$ and $l_{y}=50 \mathrm{~mm}$ for the second. Finally, if $l_{x} \gg l_{y}$ the angle approaches $55^{\circ}$.

The same trend is observed for the angular frequency ( $\omega=2 \pi f$, where $f$ is the frequency) and for the modulus of the wave number: in the case $l_{x}=100 \mathrm{~mm}$ and variable $l_{y}$, $|\vec{k}|$ decreases from 2.3 to $1.5 \mathrm{~mm}^{-1}$ with increasing $l_{y}$ [Fig. 7(b)], and $w$ also decreases from 2.75 to $1.46 \mathrm{~Hz}$ [Fig. 7(c)]. In addition, as $l_{y}$ is increased, the value of the component $k_{x}$ changes from $2 \mathrm{~mm}^{-1}$ to $1.1 \mathrm{~mm}^{-1}$, for $l_{x}=100 \mathrm{~mm}$, and the component $k_{y}$ decreases from $2 \mathrm{~mm}^{-1}$ to $0.94 \mathrm{~mm}^{-1}$.

The dispersion relations $\omega\left(k_{x, y}\right)$ have been obtained in the $x$ and $y$ directions from spatiotemporal diagrams, as explained in Ref. [9]. The group velocity $\vec{v}_{\mathrm{gr}}=\left(\partial \omega / \partial k_{x}\right) \hat{x}$ $+\left(\partial \omega / \partial k_{y}\right) \hat{y}$ can be calculated, and compared to the phase velocity $\vec{v}_{\mathrm{ph}}$. For the considered waves, we found $v_{x}^{\mathrm{gr}} \sim v_{x}^{\mathrm{ph}}$ and $v_{y}^{\mathrm{gr}} \sim \frac{2}{3} v_{y}^{\mathrm{ph}}$. This result confirms that the wave trains propagate from the cold to the hot end walls of the cell and that the waves originate in a source at the center of the cell [21] and not at the side walls.

This experiment is a good system to study the effects of a convective instability in finite systems and the transition to an absolute one. Similar experiments currently under way [17] show that this effect can be responsible for the generation of wave packets in the $y$ direction (wall modes [18]).

\section{B. Local Marangoni number}

If the temperature difference of Fig. 5 is fitted by a straight line, the value of the average gradient $\left(T_{+}\right.$ $\left.-T_{-}\right) / l_{x}$ and the corresponding Marangoni number (Ma $\simeq 473$ ) are found. This value is larger than the theoretical one $(\mathrm{Ma} \simeq 350)$ for this experimental configuration [2]. We presume that the difference is due mainly to the influence of the end walls, which leads to a nonlinear temperature profile.

Nevertheless, if the average temperature gradient is taken to calculate the Ma number, it amounts to consider the gradient constant along $x$. To show that this is not the case, a thermocouple is introduced in the fluid at $z=0.5 \mathrm{~mm}$ from the bottom and moved along the gradient direction taking the temperature at even distances $(1 \mathrm{~mm})$. We can then draw the horizontal temperature profile [Fig. 3(a)]. As said before, the temperature is nearly constant along the $y$ direction, so the temperature gradient can be calculated at every point $x_{i}$ as $\beta_{L}\left(x_{i}\right)=\partial T /\left.\partial x\right|_{x=x_{i}}$. As we stated, the temperature profile remains constant for different depths $z$, and the local temperature gradient is $z$-independent. From the horizontal profile of Fig. 3(a), a local Marangoni number $\mathrm{Ma}_{L}$ is calculated at each point,

$$
\mathrm{Ma}_{L}=\frac{\left|\frac{\partial \sigma}{\partial T}\right| \beta_{L} h^{2}}{\rho \nu \kappa},
$$

where $\beta_{L}=|d T / d x|$ and $d x=1 \mathrm{~mm}$.

The result is shown in Fig. 8 for $l_{x}=100 \mathrm{~mm}$ and $l_{y}$ $=100 \mathrm{~mm}$. Close to both sides there exists a region of 10 $\mathrm{mm}$ that corresponds to the meniscus and the region where the flow is no longer parallel to the surface (vertical dashed lines).

It is interesting to note that the value $\mathrm{Ma}_{L}=350 \pm 40$ (straight line) defines a zone near the hot side where waves 


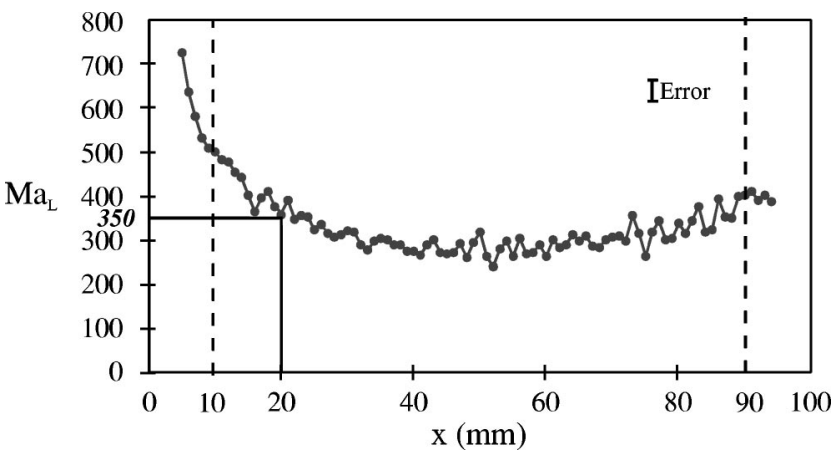

FIG. 8. Local Marangoni number calculated from the profile of Fig. 3(a) for $l_{x}=100 \mathrm{~mm}$ and $l_{y}=100 \mathrm{~mm}$. For $x<10 \mathrm{~mm}$ and $x$ $>90 \mathrm{~mm}$ (dotted vertical lines), meniscus effects are strong. For $\mathrm{Ma}_{L} \geqslant 350$, waves show up and propagate towards $T_{+}$.

appear: they approximately fill $10 \mathrm{~mm}$ up to the beginning of the meniscus zone. Thus, it can be said that $\mathrm{Ma}_{L} \simeq 350$ is a critical value above which waves appear in the container. The horizontal temperature profiles at the surface obtained with the infrared sensor produce the same value of $\mathrm{Ma}_{L}$.

This result prompted us to think that the right way to obtain the Ma number is to measure it locally. According to this, if $\Delta T$ is increased, the curve of $\mathrm{Ma}_{L}$ raises up and as a consequence the zone of waves increases in size because there is a larger zone of the fluid whose $\mathrm{Ma}_{L}$ is bigger than the critical one. For a $\Delta T$ big enough, waves fill the whole container. This can explain why usually the critical Ma number found in most of the experiments differs from the one calculated theoretically.

\section{Amplitude of the waves}

According to the shadowgraph images, hydrothermal waves appear near the hot side at some distance from the wall. In order to study the instability, it is interesting to know how the amplitude varies along $x$. As an example, we present the results corresponding to $\Delta T=11.9^{\circ} \mathrm{C}$, where the waves are present in a small $x-y$ region of the container. The horizontal dimensions are $l_{x}=100 \mathrm{~mm}$ and $l_{y}=100 \mathrm{~mm}$. We measured the temperature from $x=2 \mathrm{~mm}$ (near the hot copper block) to $x=28 \mathrm{~mm}$ (towards the cold side) at each millimeter, inside the region where waves are seen. At each point, the temporal evolution of the temperature is taken during several minutes. A Fourier analysis determines the frequency and the amplitude for each $x$ (Fig. 9). In the region $x>20 \mathrm{~mm}$, there are no waves. For $12<x \leqslant 20$ the amplitude

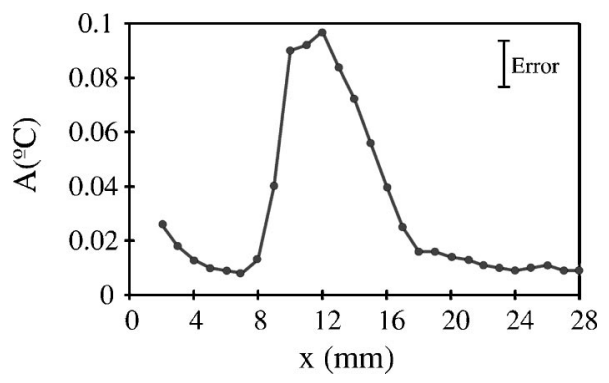

FIG. 9. Peak-to-peak amplitude vs $x$, for $\Delta T=11.9^{\circ} \mathrm{C}$ and $l_{x}$ $=100 \mathrm{~mm}, l_{y}=100 \mathrm{~mm}$. The maximum of the temperature disturbances is located near the hot side $\left(T_{+}\right)$and waves travel from right to left. This result agrees with that presented in Fig. 6 of Ref. [15]. increases as $x$ decreases, with a maximum of the amplitude located at a distance of $x=12 \mathrm{~mm}$, near the place where the meniscus becomes important. For $8<x \leqslant 12$ the amplitude decreases with $x$ decreasing, and finally vanishes for $x<8$. We have tried to find a relation between the temperature amplitude of the waves, $A(x)$, and the distance to the threshold at each point: $\epsilon(x)=\left[\mathrm{Ma}_{L}(x)-\mathrm{Ma}_{c}\right] / \mathrm{Ma}_{c}$. In particular, we tried to find a relation of the type $A=\epsilon^{1 / 2}$ but the experimental resolution was not high enough because a precision of $\mathrm{mK} / \mathrm{mm}$ in the determination of the temperature gradient is necessary to distinguish between various laws. Future work will be done to obtain this relation.

If we compare this result with the numerical simulations of $\mathrm{Xu}$ and Zebib [15] in a fluid with $\mathrm{Pr}=10$, the same conclusion is found: waves travel from right $\left(T_{-}\right)$to left $\left(T_{+}\right)$ filling a zone near the hot side. The amplitude is maximum near the hot side and decreases towards the cold side until waves disappear. Obviously, this result is in contrast with the theoretical one [2-6] in which waves appear filling the whole container at the threshold. This is due to the fact that the Ma number is not uniform in a finite fluid layer.

\section{CONCLUSIONS}

The influence of the container geometry in the convection of a laterally heated fluid layer has been studied in the case of an intermediate Pr number, showing the dependence of some physical properties of the waves (frequency, wave number, angle of propagation). The influence of the lateral confinement on the threshold has also been established quantitatively. In particular, a shorter $l_{y}$ gives rise to a higher threshold as well as a bigger frequency and wave number. We have reproduced the results of previous experimental works for the cases $l_{x} \simeq l_{y}[13,14]$ and $l_{x} \ll l_{y}[16]$, although in some experiments the confinement causes a different angle [7].

In the $y$ direction the dynamics has homogeneous experimental parameters and so the supercritical bifurcation can be described by a complex Ginzburg-Landau equation. The components of the group velocity $\left(v_{x}^{\mathrm{gr}}, v_{y}^{\mathrm{gr}}\right)$, the correlation length $\left(\xi_{0}\right)$, and the distance from the threshold $(\epsilon)$ are determined for this experiment.

We have shown that the right way to obtain the Marangoni number is to measure it locally. Hydrothermal waves arise when a critical $\mathrm{Ma}_{L}=350$ is reached and they expand in the gradient direction over a region near the hot side. As $\mathrm{Ma}_{L}$ increases, the zone where waves exist grows and eventually the entire layer is filled with them.

The amplitude of the waves decreases as $x$ increases showing a maximum at $12 \mathrm{~mm}$ from the hot side. This result is in agreement with recent numerical simulations [15].

\section{ACKNOWLEDGMENTS}

We are grateful to Héctor Mancini and Diego Maza for their comments. We also thank François Daviaud and his group for their valuable help, DGICYT (Spanish Government) for financing through Project PB95-0578, and Universidad de Navarra (Project PIUNA). M.A.P. acknowledges a grant from the Asociación de Amigos de la Universidad de Navarra. J.B. acknowledges a grant from SEUID (Spanish Government). 
[1] N. Mukolobwiez, A. Chiffaudel, and F. Daviaud, Phys. Rev. Lett. 80, 4661 (1998).

[2] M.K. Smith and S.H. Davis, J. Fluid Mech. 132, 119 (1983).

[3] M.K. Smith, Phys. Fluids 29, 3182 (1986).

[4] G.Z. Gershuni, P. Laure, V.M. Myznikov, B. Roux, and E.M. Zhukhovitsky, Microgravity Q. 2, 141 (1992).

[5] P.M. Parmentier, V.C. Regnier, and G. Lebon, Int. J. Heat Mass Transf. 36, 2417 (1993).

[6] J.F. Mercier and C. Normand, Phys. Fluids 8, 1433 (1996).

[7] F. Daviaud and J.M. Vince, Phys. Rev. E 48, 4432 (1993).

[8] A.B. Ezersky, A. Garcimartín, H.L. Mancini, and C. PérezGarcía, Phys. Rev. E 48, 4414 (1993).

[9] J. Burguete, H. Chaté, F. Daviaud, and N. Mukolobwiez, Phys. Rev. Lett. 82, 3252 (1999).

[10] A. Garcimartín, N. Mukolobwiez, and F. Daviaud, Phys. Rev. E 56, 1699 (1997).

[11] Y. Kamotani, J.H. Lee, S. Ostrach, and A. Pline, Phys. Fluids
A 4, 955 (1992).

[12] D. Schwabe, U. Möller, J. Schneider, and A. Scharmann, Phys. Fluids A 4, 2368 (1992).

[13] M.A. Pelacho and J. Burguete, Phys. Rev. E 59, 835 (1999).

[14] R.J. Riley and G.P. Neitzel, J. Fluid Mech. 359, 143 (1998).

[15] J. Xu and A. Zebib, J. Fluid Mech. 364, 187 (1998).

[16] J. Burguete, N. Mukolobwiez, F. Daviaud, N. Garnier, and A. Chiffaudel (unpublished).

[17] N. Garnier and A. Chiffaudel (private communication).

[18] S.M. Tobias, M.R.E. Proctor, and E. Knobloch, Physica D 113, 43 (1998).

[19] R.J. Deissler, J. Stat. Phys. 40, 371 (1985).

[20] P. Manneville, Dissipative Structures and Weak Turbulence (Academic Press, San Diego, 1990).

[21] M. van Hecke, C. Storm, and W. van Saarloos, Physica D 134, 1 (1999). 